\title{
Prediction of optimal debulking surgery in ovarian cancer
}

\author{
Yong Jung Song ${ }^{1,2}$ \\ ${ }^{1}$ Department of Obstetrics and Gynecology, Pusan National University Yangsan Hospital, Yangsan, South Korea; ${ }^{2}$ Research Institute for \\ Convergence of Biomedical Science and Technology, Pusan National University Yangsan Hospital, Pusan National University School of Medicine, \\ Yangsan, South Korea \\ Correspondence to: Yong Jung Song, MD. Department of Obstetrics and Gynecology, Pusan National University Yangsan Hospital, Yangsan, South \\ Korea; Research Institute for Convergence of Biomedical Science and Technology, Pusan National University Yangsan Hospital, Pusan National \\ University School of Medicine, 20, Geumo-ro, Mulgeum-eup, Yangsan-si, Gyeongsangnam-do, 50612, South Korea. Email: gynsong@gmail.com.
}

\begin{abstract}
The mainstay management of advanced ovarian cancer is maximal cytoreductive surgery followed by chemotherapy. Neoadjuvant chemotherapy (NACT) and interval debulking surgery (IDS) are alternative treatments for patients with comorbidity, poor performance status, and predicted for suboptimal debulking surgery. It is the invariable principle in any situation that no residual disease after the completion of surgery is useful for patients with ovarian cancer. Therefore, the prediction of optimal debulking before the treatment of ovarian cancer is of utmost importance. Many studies have reported on the use of serum biomarkers, such as cancer antigen 125 (CA125) or human epididymis 4 (HE4), and imaging studies, such as computed tomography (CT), diffusion-weighted magnetic resonance imaging (DW-MRI), and positron emission tomography (PET)/CT, to identify adequate surgical candidates for primary debulking surgery (PDS). Laparoscopy has also been studied as a reliable tool for the prediction of optimal debulking. Here, we summarize a review of the related literature.
\end{abstract}

Keywords! Ovarian cancer; optimal cytoreduction; prediction

Submitted Feb 14, 2020. Accepted for publication Jul 01, 2020.

doi: $10.21037 / g s-2019$-ursoc-08

View this article at: http://dx.doi.org/10.21037/gs-2019-ursoc-08

\section{Introduction}

Advanced ovarian cancer is the second most common female genital malignancy globally, with a 5 -year survival rate ranging from $30 \%$ to $50 \%$ (1). Primary debulking surgery (PDS) and taxane-platinum combination chemotherapy are standard methods of management of epithelial ovarian cancer (EOC) (2,3). Many studies have reported that the significant prognostic factor for survival is the size of postoperative residual disease (4-6). Generally, optimal cytoreduction has been defined as the largest diameter of residual disease of less than $1 \mathrm{~cm}$ (3). Currently, no macroscopic residual disease (R0 resection) has incremental benefits over residual disease under $1 \mathrm{~cm} \mathrm{(7).}$ Suboptimal surgery has a negative effect on survival, so treatment strategies to avoid unnecessary surgery should be considered $(2,8)$. If complete cytoreduction is considered impossible or has unacceptable preoperative morbidity, neoadjuvant chemotherapy (NACT) followed by interval debulking surgery (IDS) could be an alternative option according to the results of two landmark phase III clinical trials [European Organization for Research and Treatment of Cancer (EORTC) 55971 and primary chemotherapy versus primary surgery for newly diagnosed advanced ovarian cancer (CHORUS) trial] $(9,10)$. If optimal debulking is predicted, PDS should be performed preferentially, but with the probability of sub-optimal debulking, NACT and IDS should be considered (11). Therefore, prediction of patients who are not feasible to achieve optimal debulking is important. Many investigations have been conducted to identify factors that most accurately predict patients who will be good candidates for optimal cytoreduction after PDS. However, there are no accurate and broadly used indications for NACT $(12,13)$. Tumor markers, hematologic indicator, radiologic images, and diagnostic laparoscopy have been applied to predict optimal 
debulking surgery in various studies. In some studies, high levels of tumor markers such as human epididymis 4 (HE4) and cancer antigen 125 (CA125) have been used as predictors of suboptimal debulking surgery $(12,14,15)$. In several studies, large amount of ascites, liver parenchymal metastases, suprarenal lymphadenopathy, porta hepatis metastases, involvement of the mesentery root of the small bowel, lesser sac involvement, and diaphragmatic disease on computed tomography (CT) scan findings were reported as predictors for suboptimal debulking $(3,16,17)$. Diagnostic laparoscopy might be useful for the direct visualization of tumor extension and more precise prediction of tumor resectability $(8,18,19)$. However, there is no consensus on accurate prediction for optimal debulking surgery in patients with ovarian cancer. Here, we present a review of studies dealing with the various criteria used to evaluate optimal debulking in advanced EOC.

\section{Tumor marker and hematologic parameters}

\section{CA125 and HE4}

One of the most studied markers used in the prediction of optimal debulking in advanced ovarian cancer is CA125. Suidan et al. reported CA125 of at least $600 \mathrm{U} / \mathrm{mL}$ as a predictive marker for suboptimal residual disease $(>1 \mathrm{~cm}$ residual) after PDS in multicenter, nonrandomized trial (16). Furthermore, a retrospective study identified that $90 \%$ reduction in preoperative CA125 level was associated with complete IDS after NACT (20). In a recent metaanalysis, the researchers made efforts to elucidate CA125 cut-off levels as a predictor of optimal debulking after PDS by integrating 14 studies with 2,192 patients. Results of preoperative serum CA125 for predicting optimal cytoreduction in advanced ovarian cancer is a low positive and high negative likelihood ratio, respectively. However, a preoperative serum CA125 level over $500 \mathrm{U} / \mathrm{mL}$ was significantly associated with suboptimal cytoreduction [odds ratio, 3.69; 95\% confidence interval (CI), 2.02-6.73] (14). HE4 is another useful biomarker that has been studied to predict optimal debulking in advanced ovarian cancer. A meta-analysis by Pergialiotis et al. reported that the pooled sensitivity and specificity of HE4 for the anticipation of optimal debulking were 0.81 (95\% CI, 0.74-0.86) and 0.80 (95\% CI, 0.75-0.84), respectively. They also showed promising results that the diagnostic odds ratio was 13.88 (95\% CI, 7.18-26.84) and area under the curve was $0.86 \pm 0.03(21)$.

\section{Hematologic predictor}

Recently, lymphocyte-monocyte ratio (LMR) was identified as a possible prognosticator for advanced ovarian cancer among hematologic inflammatory markers. Although mechanisms underlying the relationship between higher LMR and optimal cytoreduction have not been fully explained, some suggestions can be derived by considering the nature of these inflammatory cells. The role of lymphocytes is to fight against cancer cells. Monocytes increase as tumor burden grows in advanced ovarian cancer (22). Eo et al. published that higher LMR was found to be the strongest predictors for optimal cytoreduction ( $\mathrm{P}=0.0015)$ in 154 patients with stage IIIIV advanced ovarian cancer (15). However, there was no consensus on the cut-off values, and prospective use of these markers for optimal debulking cytoreduction in advanced ovarian cancer is under investigation. Furthermore, factors of performance and nutritional status, such as age, race, smoking status, creatinine, and albumin levels, have also been elucidated with respect to patient selection for NACT and IDS, taking into account the postoperative morbidity $(23,24)$.

\section{Preoperative images}

\section{$C T$}

Preoperative imaging such as CT can provide crucial information about the location and extent of tumor. Gynecologic oncologist may predict optimal debulking with this information. Many investigators have studied the predicting capability for optimal debulking before PDS in patients with advanced ovarian cancer. Image-based models for the prediction of optimal debulking are summarized in Table 1. Bristow et al. reported that peritoneal thickening or implants ( $\geq 2 \mathrm{~cm}$ ); involvement of the spleen, stomach, or lesser sac; bowel mesenteric extension $(\geq 2 \mathrm{~cm})$; suprarenal paraaortic lymph nodes enlargement $(\geq 1 \mathrm{~cm})$; and pelvic sidewall involvement and/or hydroureter were the most important predictive factors for suboptimal debulking. They proposed a unique predictive index score (PIS), which is figured by above-mentioned factors. Over PIS 4 had the highest overall accuracy at $92.7 \%$ and identified patients undergoing suboptimal debulking with a sensitivity of $100 \%(21 / 21)$. The specificity, or capability to identify patients undergoing optimal debulking, was $85.0 \%$ (17/20). The positive predictive values (PPVs) and negative predictive values (NPVs) of a PIS $\geq 4$ were $87.5 \%$ (21/24) 
Table 1 Models constructed by image to predict the optimal cytoreduction in patients with advanced ovarian cancer

\begin{tabular}{|c|c|c|c|c|}
\hline First author & Modality & $\begin{array}{l}\text { Patients } \\
\text { (No.) }\end{array}$ & Findings included in the model & $\begin{array}{l}\text { Prediction model for suboptimal } \\
\text { cytoreduction }\end{array}$ \\
\hline \multirow[t]{11}{*}{ Bristow (25) } & CT & 42 & 2 points & Index score cutoff $\geq 4$ \\
\hline & & & Peritoneal thickening & PPV $87.5 \%$ \\
\hline & & & Peritoneal implants $\geq 2 \mathrm{~cm}$ & NPV $100 \%$ \\
\hline & & & $\begin{array}{l}\text { Omental involvement to stomach, spleen, } \\
\text { or lesser sac }\end{array}$ & \\
\hline & & & $\begin{array}{l}\text { Extension to pelvic sidewall, parametrium, } \\
\text { or ureter }\end{array}$ & \\
\hline & & & Performance status $\geq 2$ & \\
\hline & & & 1 point & \\
\hline & & & $\begin{array}{l}\text { Diaphragm or lung bars involvement } \geq 2 \mathrm{~cm} \text {, } \\
\text { or confluent plaque }\end{array}$ & \\
\hline & & & Inguinal canal disease or lymph nodes $\geq 2 \mathrm{~cm}$ & \\
\hline & & & $\begin{array}{l}\text { Liver metastases } \geq 2 \mathrm{~cm} \text { on surface, or } \\
\text { parenchymal lesion (any size) }\end{array}$ & \\
\hline & & & $\begin{array}{l}\text { Porta hepatis or gallbladder fossa disease } \\
\geq 1 \mathrm{~cm}\end{array}$ & \\
\hline \multirow{7}{*}{ Suidan (16) } & & & 2 points & \\
\hline & & & Age $\geq 60$ years & \\
\hline & & & $\mathrm{CA} 125 \geq 500 \mathrm{U} / \mathrm{mL}$ & \\
\hline & & & $\begin{array}{l}\text { Suprarenal, supradiaphragmatic lymph node } \\
>1 \mathrm{~cm}\end{array}$ & \\
\hline & & & Diffuse small bowel adhesion/thickening & \\
\hline & & & Perisplenic lesion $>1 \mathrm{~cm}$ & \\
\hline & & & Root of superior mesenteric artery lesion $>1 \mathrm{~cm}$ & \\
\hline \multirow[t]{3}{*}{ Janco (17) } & CT & 279 & ECOG performance status $\geq 2$ & $\begin{array}{l}\text { Independent predictor by multivariate } \\
\text { analysis }\end{array}$ \\
\hline & & & Diffuse peritoneal thickening & \\
\hline & & & Lymphadenopathy & \\
\hline
\end{tabular}

Table 1 (Continued) 
Table 1 (Continued)

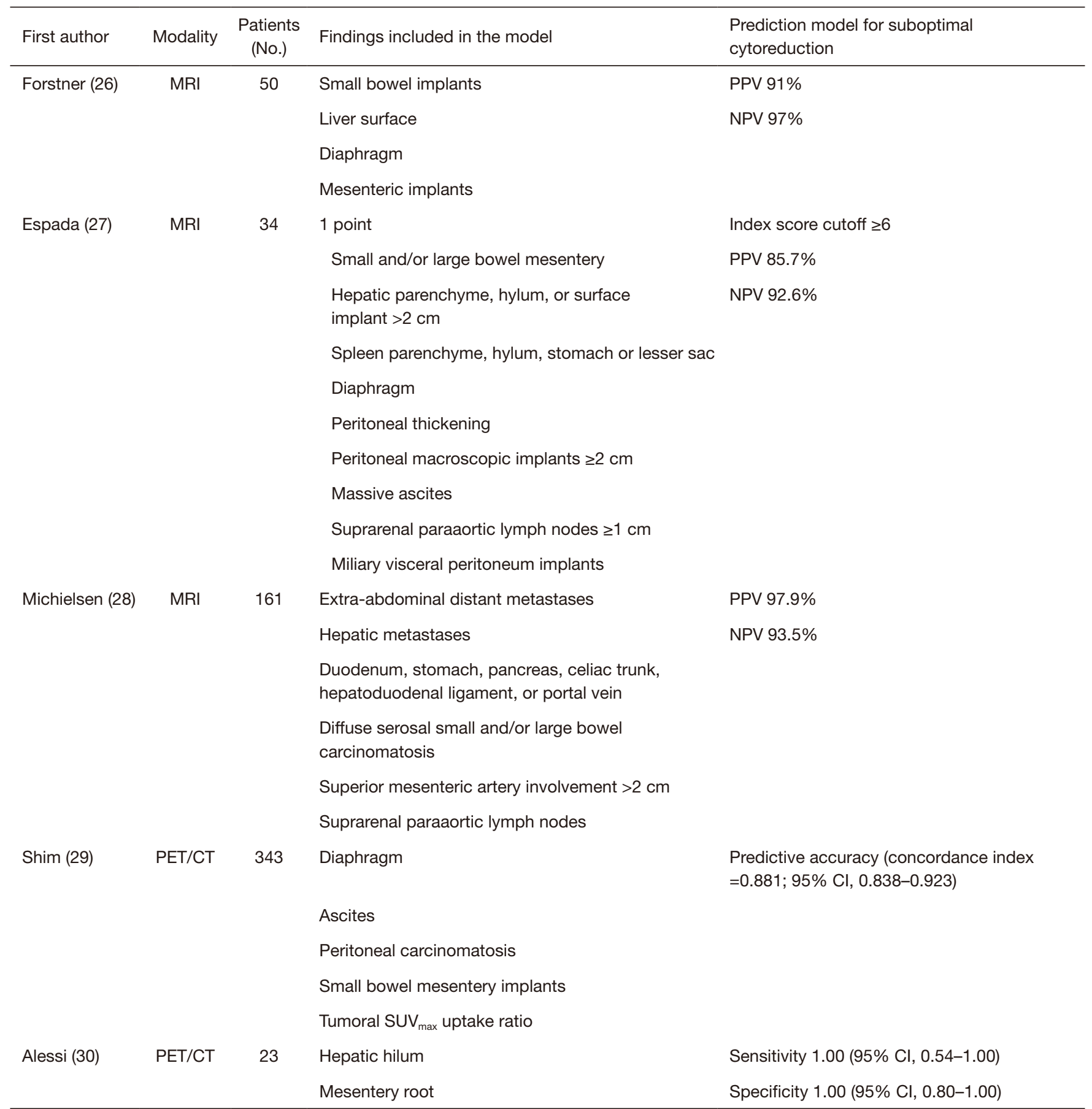

CT, computed tomography; PPV, positive predictive value; NPV, negative predictive value; ASA, American Society of Anesthesiologists; CA125, cancer antigen 125; ECOG, Eastern Cooperative Oncology Group; MRI, magnetic resonance imaging; PET, positron emission tomography; $\mathrm{Cl}$, confidence interval; SUV, standardized uptake value. 
and $100 \%$, respectively (25). In multivariate analysis by Janco et al., no ascites, omental involvement, and diffuse peritoneal thickening on CT were independently associated with optimal debulking. They also developed nomogram as a predictive model by combining age and performance status. For instance, if a 50-year old patient presents with ascites and diffuse peritoneal thickening on preoperative CT, the predicted probability of complete cytoreduction is approximately $27 \%$, with a sensitivity and specificity of $84 \%$ and $43 \%$, respectively (17). However, a systematic review to evaluate CT-based prediction models for optimal debulking in advanced ovarian cancer concluded that there are few studies externally validated with a high predictive value (11).

\section{Magnetic resonance imaging (MRI)}

Generally, diagnostic imaging is predominantly based on CT before surgery in advanced ovarian cancer. Unfortunately, this preoperative evaluation is incomplete as small tumor deposits can be missed and distinguishing malignant from benign tissue can be difficult. MRI has good image contrast of soft tissue and shows a detailed view of the structures and its position toward the surrounding tissue. Forstner et al. reported that the sensitivity and specificity of conventional MRI in predicting suboptimal debulking were 0.91 (95\% CI, 0.59-1.0) and 0.97 (95\% CI, $0.87-1.0)$, respectively. On the other hand, the sensitivity and specificity of CT for predicting suboptimal debulking were 0.50 (95\% CI, 0.12-0.88) and 1.0 (95\% CI, $0.91-$ 1.0), respectively (26). A retrospective analysis by Espada et al. published that diffusion-weighted MRI (DW-MRI) precisely predicts optimal debulking in $91 \%$ of patients (31/34) using predictive score $>6(27)$. The authors asserted the superiority of DW-MRI over CT due to better contrast resolution resulting in improved detection of sites that are critical for surgery, such as intestinal serosal metastases, central mesenteric vessel metastases, and unresectable distant metastases. Michielsen et al. made a comparison between DW-MRI and CT for their diagnostic accuracy. PDS was performed in 44 of 94 patients, and suboptimal debulking $(\geq 1 \mathrm{~cm})$ was performed in 39 women $(89 \%)$. In this analysis, the sensitivity and specificity for predicting suboptimal debulking (with residual disease of any size) of DW-MRI were 0.94 (95\% CI, 0.83-0.99) and 0.98 (95\% CI, 0.88-1.00), respectively. For CT, the sensitivity and specificity for predicting suboptimal debulking were 0.66 (95\% CI, 0.52-0.78) and 0.77 (95\% CI, 0.63-0.87), respectively (28).

\section{Positron emission tomography (PET)/CT}

PET/CT detects enhanced glucose metabolism of cancer cells, so it suggests valuable information on tumor extension, especially useful for the identification of distant metastases. A prospective study used a prediction model including five PET/CT features and a surgical aggressiveness index to predict suboptimal debulking with residual disease of any size in 343 women with advanced ovarian cancer (29). The authors identified several PET/CT factors that were independently associated with suboptimal debulking, such as diaphragmatic disease and small bowel mesentery metastases. The sensitivity and specificity of PET/CT for suboptimal debulking (with residual disease of any size) were 0.66 (95\% CI, 0.60-0.73) and 0.88 (95\% CI, 0.80 $0.93)$, respectively. Alessi et al. reported that the sensitivity and specificity of PET/CT for evaluating incomplete debulking (with residual disease of any size) were 1.00 (95\% CI, 0.54-1.00) and 1.00 (95\% CI, 0.80-1.00) (30).

\section{Combination preoperative image with tumor marker}

A prospective trial of preoperative $\mathrm{CT}$ in combination with serum CA125 by Suidan et al. was conducted to calculate the rates of suboptimal PDS ( $\geq 1 \mathrm{~cm}$ residual disease) in patients with advanced ovarian cancer. Of 350 patients, 261 had optimal debulking and the remaining 89 had suboptimal debulking (31). The following criteria were independently associated with suboptimal debulking: age $\geq 60$ years, CA125 level $\geq 500 \mathrm{U} / \mathrm{mL}$, American Society of Anesthesiologists Physical Status (ASA) score 3 or 4, suprarenal lymphadenopathy (incorporating cardiophrenic) $>1 \mathrm{~cm}$, diffuse small bowel adhesions/thickening, spleen lesion $>1 \mathrm{~cm}$, small bowel mesentery extension $>1 \mathrm{~cm}$, involvement in the root of the superior mesenteric artery $>1 \mathrm{~cm}$, and lesser sac metastasis $>1 \mathrm{~cm}$. In a retrospective study of 129 patients with advanced ovarian cancer, Arab et al. demonstrated that serum CA125 $>420 \mathrm{U} / \mathrm{mL}$, massive ascites, and liver metastasis are powerful predictive factors for suboptimal debulking in PDS (32).

\section{Diagnostic laparoscopy}

The rationale for a laparoscopic evaluation before PDS includes (I) this surgical concept could avoid a useless laparotomy, which has no survival benefits due to suboptimal debulking; (II) patients not considered for 
optimal debulking could proceed immediately to NACT; and (III) pathologic diagnosis and molecular profiling are possible. A study performed by Fagotti et al., in which clinicradiological factors were collected preoperatively and all patients were submitted to both laparoscopy and laparotomy sequentially (33), assessed items during laparoscopy including the bilaterality of ovarian masses, peritoneal and diaphragmatic carcinomatosis, omental cake or nodules, mesenteric retraction, bowel and stomach extension, liver involvements, and bulky lymph node enlargement. After completing laparoscopy, the surgeon stated the probability that optimal debulking was possible was based on the absence of the conventional criteria of unresectability, which were extensive bulky peritoneal carcinomatosis, porta hepatis involvement, retraction of the bowel mesentery, diaphragm bulky disease, and/or unresectable upper abdominal disease (34). Optimal debulking was completed in $87 \%$ of patients $(34 / 39)$ who had favorable laparoscopic findings. They showed that the overall accuracy rate of laparoscopy was $90 \%$ for predicting optimal debulking. The NPVs of clinico-radiological evaluation and laparoscopy were $73 \%$ and $100 \%$, respectively, and the PPVs were both $87 \%$. Based on the above study, Fagotti et al. extended their laparoscopic evaluation trial (18) and showed prospective data of 113 patients who underwent laparoscopy. They used the predictive index value (PIV) score for investigating the probability of optimal debulking. The individual items were added up to obtain an overall score (33). The overall accuracy of the laparoscopy-based score ranged from $77 \%$ to $100 \%$ in predicting optimal debulking. The results confirmed that at a PIV of $\geq 8$, the probability of debulking optimally (residual tumor $\leq 1 \mathrm{~cm}$ ) at laparotomy was 0 . The role of diagnostic laparoscopy in predicting for $\mathrm{R} 0$ resection in advanced ovarian cancer was also evaluated by other researcher (35). In this study, diagnostic laparoscopy was performed in 87 patients. Candidates for R0 resection were $61 \%$ (53/87 patients) and, therefore, they performed PDS. The optimal debulking rate in this group was $96 \%$. There were no major perioperative morbidity and mortality related to laparoscopy. Brun et al. conducted the external validation of using the Fagotti criteria in a cohort of 55 patients with stage III-IV ovarian cancer (36). Of the 55 patients, 26 underwent primary PDS after diagnostic laparoscopy, and the remaining 29 were treated with NACT. A PIV of $\geq 8$ was associated with suboptimal cytoreduction. The sensitivity, specificity, PPV, NPV, and accuracy values were $46 \%, 89 \%, 89 \%, 44 \%$, and $60 \%$, respectively. The Fagotti group prospectively evaluated the learning curve for determining the PIV. This study revealed laparoscopic-based scores of gynecologic oncologic fellows with at least 12 months experience similar to those of senior surgeons (37). Another group in Dutch published the laparoscopy to predict the result of primary cytoreductive surgery in advanced ovarian cancer patients (LAPOVCA) randomized clinical trial (38). They used the following criteria for prediction of suboptimal cytoreduction: extensive agglutinated intra-abdominal metastatic disease (including spleen or retrohepatic area involvement), extensive serosa invasion of the bowel and/or mesenteric involvement (the possibility of multiple bowel resections), and extensive (unresectable) peritoneal carcinomatosis at the subdiaphragm. Using these laparoscopic finding, futile laparotomy so called "suboptimal cytoreduction" appeared in $10 \%$ (10/102 patients) in the laparoscopy group versus $39 \%$ (39/99 patients) in the primary surgery group (relative risk, $0.25 ; 95 \%$ CI, 0.13-0.47; $\mathrm{P}<0.001)$. Diagnostic laparoscopy before surgery decreases the number of futile laparotomies in patients with advanced ovarian cancer. The accuracy of diagnostic laparoscopy for predicting optimal debulking in patients with advanced ovarian cancer was evaluated in a recent Cochrane Review. The authors concluded that laparoscopy might be a useful diagnostic tool for predicting the residual disease after PDS. Therefore, the selection of women who would benefit from PDS may be possible. However, due to the large heterogeneity of the included studies, careful interpretation of the study result is crucial (39). The laparoscopic criteria for the prediction of optimal debulking are summarized in Table 2.

\section{Conclusions}

Basic treatment of patients with EOC is optimal cytoreduction with acceptable morbidity followed by platinum- and taxane-based chemotherapy. NACT may decrease the morbidity at the time of IDS; however, it does not improve survival. So, prediction for optimal cytoreduction is very important for not performing futile surgery. We reviewed that preoperative serum levels of CA125 and HE4 are useful biomarkers for optimal cytoreduction and another hematologic marker using lymphocyte-monocyte is being investigated. Imaging studies using CT, DW-MRI, and PET/CT are also valuable for the preoperative evaluation of optimal debulking. However, that these factors were investigated in a retrospective manner is a limitation. There has been no prospective study yet, and there is no competent method for predicting optimal 
Table 2 Laparoscopic criteria for suboptimal cytoreduction in patients with ovarian cancer

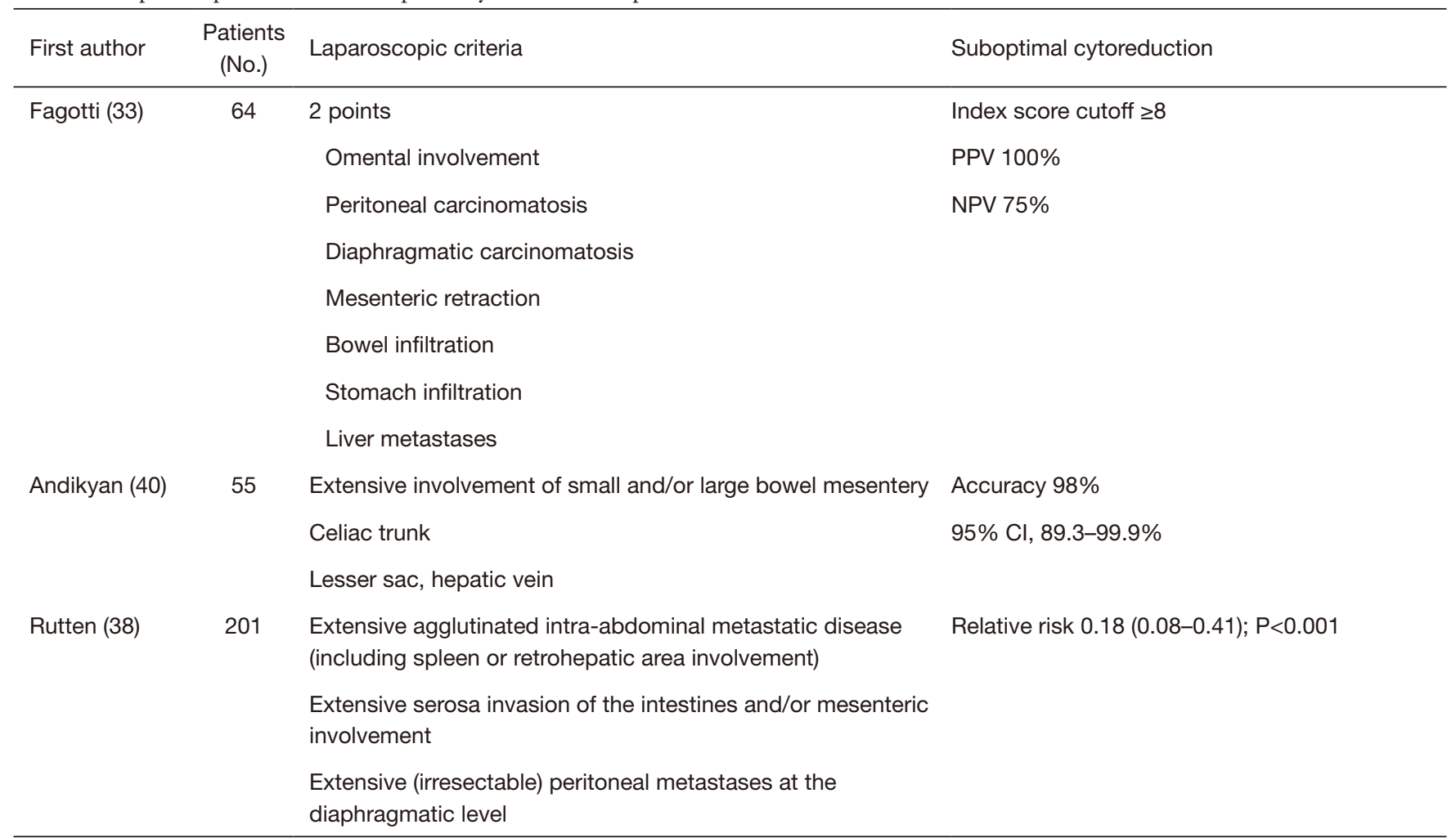

PPV, positive predictive value; NPV, negative predictive value; $\mathrm{Cl}$, confidence interval.

cytoreduction. Large-scale randomized clinical trials of laparoscopic evaluation using the scoring system showed that it may be a useful tool. Therefore, an effort should be made to select patients with optimal cytoreduction prognoses using multiple methods, such as serum biomarkers, imaging studies, and diagnostic laparoscopy, and having discussions with multidisciplinary team to yield more results from large clinical trials.

\section{Acknowledgments}

Funding: This study was supported by a 2020 research grant from Pusan National University Yangsan Hospital.

\section{Footnote}

Provenance and Peer Review: This article was commissioned by the Guest Editors (Sang Yoon Park, Jae Weon Kim) for the series "Ultra-Radical Surgery in Ovarian Cancer: Surgical Techniques for Gynecologic Oncologist” published in Gland Surgery. The article was sent for external peer review organized by the Guest Editors and the editorial office.

Conflicts of Interest: The author has completed the ICMJE uniform disclosure form (available at http://dx.doi. org/10.21037/gs-2019-ursoc-08). The series "UltraRadical Surgery in Ovarian Cancer: Surgical Techniques for Gynecologic Oncologist" was commissioned by the editorial office without any funding or sponsorship. The author has no other conflicts of interest to declare.

Ethical Statement: The author is accountable for all aspects of the work in ensuring that questions related to the accuracy or integrity of any part of the work are appropriately investigated and resolved.

Open Access Statement: This is an Open Access article distributed in accordance with the Creative Commons Attribution-NonCommercial-NoDerivs 4.0 International License (CC BY-NC-ND 4.0), which permits the noncommercial replication and distribution of the article with 
the strict proviso that no changes or edits are made and the original work is properly cited (including links to both the formal publication through the relevant DOI and the license). See: https://creativecommons.org/licenses/by-nc-nd/4.0/.

\section{References}

1. Siegel RL, Miller KD, Jemal A. Cancer Statistics, 2017. CA Cancer J Clin 2017;67:7-30.

2. Chi DS, Eisenhauer EL, Zivanovic O, et al. Improved progression-free and overall survival in advanced ovarian cancer as a result of a change in surgical paradigm. Gynecol Oncol 2009;114:26-31.

3. Salani R, Axtell A, Gerardi M, et al. Limited utility of conventional criteria for predicting unresectable disease in patients with advanced stage epithelial ovarian cancer. Gynecol Oncol 2008;108:271-5.

4. Chi DS, Eisenhauer EL, Lang J, et al. What is the optimal goal of primary cytoreductive surgery for bulky stage IIIC epithelial ovarian carcinoma (EOC)? Gynecol Oncol 2006;103:559-64.

5. Bristow RE, Tomacruz RS, Armstrong DK, et al. Survival effect of maximal cytoreductive surgery for advanced ovarian carcinoma during the platinum era: a metaanalysis. J Clin Oncol 2002;20:1248-59.

6. Eisenhauer EL, Abu-Rustum NR, Sonoda Y, et al. The addition of extensive upper abdominal surgery to achieve optimal cytoreduction improves survival in patients with stages IIIC-IV epithelial ovarian cancer. Gynecol Oncol 2006;103:1083-90.

7. Chang SJ, Hodeib M, Chang J, et al. Survival impact of complete cytoreduction to no gross residual disease for advanced-stage ovarian cancer: a meta-analysis. Gynecol Oncol 2013;130:493-8.

8. Fagotti A, Ferrandina G, Fanfani F, et al. A laparoscopybased score to predict surgical outcome in patients with advanced ovarian carcinoma: a pilot study. Ann Surg Oncol 2006;13:1156-61.

9. Vergote I, Trope CG, Amant F, et al. Neoadjuvant chemotherapy or primary surgery in stage IIIC or IV ovarian cancer. N Engl J Med 2010;363:943-53.

10. Kehoe S, Hook J, Nankivell M, et al. Primary chemotherapy versus primary surgery for newly diagnosed advanced ovarian cancer (CHORUS): an open-label, randomised, controlled, non-inferiority trial. Lancet 2015;386:249-57.

11. Rutten MJ, van de Vrie R, Bruining A, et al. Predicting surgical outcome in patients with International Federation of Gynecology and Obstetrics stage III or IV ovarian cancer using computed tomography: a systematic review of prediction models. Int J Gynecol Cancer 2015;25:407-15.

12. Karlsen MA, Fago-Olsen C, Hogdall E, et al. A novel index for preoperative, non-invasive prediction of macroradical primary surgery in patients with stage IIIC-IV ovarian cancer-a part of the Danish prospective pelvic mass study. Tumour Biol 2016;37:12619-26.

13. Stuart GC, Kitchener H, Bacon M, et al. 2010 Gynecologic Cancer InterGroup (GCIG) consensus statement on clinical trials in ovarian cancer: report from the Fourth Ovarian Cancer Consensus Conference. Int J Gynecol Cancer 2011;21:750-5.

14. Kang S, Kim TJ, Nam BH, et al. Preoperative serum CA125 levels and risk of suboptimal cytoreduction in ovarian cancer: a meta-analysis. J Surg Oncol 2010;101:13-7.

15. Eo W, Kim HB, Lee YJ, et al. Preoperative lymphocytemonocyte ratio is a predictor of suboptimal cytoreduction in stage III-IV epithelial ovarian cancer. J Cancer 2016;7:1772-9.

16. Suidan RS, Ramirez PT, Sarasohn DM, et al. A multicenter assessment of the ability of preoperative computed tomography scan and CA-125 to predict gross residual disease at primary debulking for advanced epithelial ovarian cancer. Gynecol Oncol 2017;145:27-31.

17. Janco JM, Glaser G, Kim B, et al. Development of a prediction model for residual disease in newly diagnosed advanced ovarian cancer. Gynecol Oncol 2015;138:70-7.

18. Fagotti A, Ferrandina G, Fanfani F, et al. Prospective validation of a laparoscopic predictive model for optimal cytoreduction in advanced ovarian carcinoma. Am J Obstet Gynecol 2008;199:642.e1-6.

19. Vizzielli G, Costantini B, Tortorella L, et al. Influence of intraperitoneal dissemination assessed by laparoscopy on prognosis of advanced ovarian cancer: an exploratory analysis of a single-institution experience. Ann Surg Oncol 2014;21:3970-7.

20. Mahdi H, Maurer KA, Nutter B, et al. The impact of percent reduction in CA-125 levels on prediction of the extent of interval cytoreduction and outcome in patients with advanced-stage cancer of Mullerian origin treated with neoadjuvant chemotherapy. Int J Gynecol Cancer 2015;25:823-9.

21. Pergialiotis V, Karampetsou N, Bellos I, et al. The diagnostic accuracy of human epididymis factor 4 for the prediction of optimal debulking in patients with ovarian cancer: a meta-analysis of observational studies. Int J Gynecol Cancer 2018;28:1471-7. 
22. Eo WK, Chang HJ, Kwon SH, et al. The lymphocytemonocyte ratio predicts patient survival and aggressiveness of ovarian cancer. J Cancer 2016;7:289-96.

23. Aletti GD, Eisenhauer EL, Santillan A, et al. Identification of patient groups at highest risk from traditional approach to ovarian cancer treatment. Gynecol Oncol 2011;120:23-8.

24. Barber EL, Rutstein S, Miller WC, et al. A preoperative personalized risk assessment calculator for elderly ovarian cancer patients undergoing primary cytoreductive surgery. Gynecol Oncol 2015;139:401-6.

25. Bristow RE, Duska LR, Lambrou NC, et al. A model for predicting surgical outcome in patients with advanced ovarian carcinoma using computed tomography. Cancer 2000;89:1532-40.

26. Forstner R, Hricak H, Occhipinti KA, et al. Ovarian cancer: staging with CT and MR imaging. Radiology 1995;197:619-26.

27. Espada M, Garcia-Flores JR, Jimenez M, et al. Diffusionweighted magnetic resonance imaging evaluation of intra-abdominal sites of implants to predict likelihood of suboptimal cytoreductive surgery in patients with ovarian carcinoma. Eur Radiol 2013;23:2636-42.

28. Michielsen K, Dresen R, Vanslembrouck R, et al. Diagnostic value of whole body diffusion-weighted MRI compared to computed tomography for pre-operative assessment of patients suspected for ovarian cancer. Eur J Cancer 2017;83:88-98.

29. Shim SH, Lee SJ, Kim SO, et al. Nomogram for predicting incomplete cytoreduction in advanced ovarian cancer patients. Gynecol Oncol 2015;136:30-6.

30. Alessi A, Martinelli F, Padovano B, et al. FDG-PET/ CT to predict optimal primary cytoreductive surgery in patients with advanced ovarian cancer: preliminary results. Tumori 2016;102:103-7.

31. Suidan RS, Ramirez PT, Sarasohn DM, et al. A multicenter prospective trial evaluating the ability of preoperative computed tomography scan and serum CA-125 to predict

Cite this article as: Song YJ. Prediction of optimal debulking surgery in ovarian cancer. Gland Surg 2021;10(3):1173-1181. doi: 10.21037/gs-2019-ursoc-08 suboptimal cytoreduction at primary debulking surgery for advanced ovarian, fallopian tube, and peritoneal cancer. Gynecol Oncol 2014;134:455-61.

32. Arab M, Jamdar F, Sadat Hosseini M, et al. Model for prediction of optimal debulking of epithelial ovarian cancer. Asian Pac J Cancer Prev 2018;19:1319-24.

33. Fagotti A, Fanfani F, Ludovisi M, et al. Role of laparoscopy to assess the chance of optimal cytoreductive surgery in advanced ovarian cancer: a pilot study. Gynecol Oncol 2005;96:729-35.

34. Fanfani F, Ferrandina G, Corrado G, et al. Impact of interval debulking surgery on clinical outcome in primary unresectable FIGO stage IIIc ovarian cancer patients. Oncology 2003;65:316-22.

35. Angioli R, Palaia I, Zullo MA, et al. Diagnostic open laparoscopy in the management of advanced ovarian cancer. Gynecol Oncol 2006;100:455-61.

36. Brun JL, Cortez A, Rouzier R, et al. Factors influencing the use and accuracy of frozen section diagnosis of epithelial ovarian tumors. Am J Obstet Gynecol 2008;199:244.e1-7.

37. Fagotti A, Vizzielli G, Costantini B, et al. Learning curve and pitfalls of a laparoscopic score to describe peritoneal carcinosis in advanced ovarian cancer. Acta Obstet Gynecol Scand 2011;90:1126-31.

38. Rutten MJ, van Meurs HS, van de Vrie R, et al. Laparoscopy to predict the result of primary cytoreductive surgery in patients with advanced ovarian cancer: a randomized controlled trial. J Clin Oncol 2017;35:613-21.

39. van de Vrie R, Rutten MJ, Asseler JD, et al. Laparoscopy for diagnosing resectability of disease in women with advanced ovarian cancer. Cochrane Database Syst Rev 2019;3:CD009786.

40. Andikyan V, Kim A, Gretz HF 3rd, et al. Laparoscopic assessment to determine the likelihood of achieving optimal cytoreduction in patients undergoing primary debulking surgery for ovarian, fallopian tube, or primary peritoneal cancer. Am J Clin Oncol 2018;41:938-42. 